\title{
VIBRATION FEEDBACKS IN PILOT-AIRCRAFT HAPTIC INTERACTION
}

\author{
Nina Malalan ${ }^{1}$, Pavel Zikmund ${ }^{2}$ \\ 1,2 Institute of Aerospace Engineering \\ Brno University of Technology \\ Technická 2, 61669 Brno, Czech Republic \\ 1 nina.malalan@vutbr.cz \\ 2 zikmund@fme.vutbr.cz
}

Keywords: haptic feedback, pilot-aircraft interaction, spatial and movement guidance, stall warning, emergency procedures

\begin{abstract}
A research about different ways of encoding the distance information with vibrotactile feedback was done as part of a bigger project with the aim of designing a device which would help the pilot to achieve greater precision during the flight. Different kinds of stall warning devices and structural additions were already designed in the field of aviation, lingering only over attentional guidance. Therefore, a lack of spatial and movement guidance was detected. This paper lingers on this research which aimed to encode and evaluate haptic guiding methods.
\end{abstract}

\section{INTRODUCTION}

It is well known that the loss of control of an aircraft represents the cause of a large number of accidents in aviation. Various EASA Annual Safety Reviews and other reference safety statistics have repeatedly identified loss of control as a primary accident category in light and heavy aircraft.

Such losses of control usually involve a full stall or an approach to a stall at some stage in the event sequence, whether as the initiating factor or as a later consequence. Even though pilots are taught to recognise, avoid and recover from stalls during the early flight training, yet the inadvertent loss of control continues to occur, with the circumstances of each new accident often similar to those of previous accidents. Different kinds of stall warning devices and structural additions were already designed, with the function of warning the pilot, postponing the stall, preventing it or making it less severe. They linger only over attentional guidance.

Therefore, a lack of spatial and movement guidance was detected. In consequence, the desire to design a device which would help the pilot to achieve greater precision during the flight appeared. A device, which would not just warn the pilot about a dangerous flight mode, but that would guide him into solving it or solving any inefficient way of flying.

As first, two vibration engines representing two directions (forward and backwards) were implemented on a joystick, which represents the control stick of an aircraft. Based on previously done work with similar tasks, a research was done to find different ways of encoding the distance information with vibrotactile feedback. The parameters, which can be manipulated, were defined and studied. Based on this study two parameters - the rhythm and the pulse's duration - were affected in three different combinations - "rhythm only", "only duration", and "rhythm \& duration". A pilot test was conducted to define their basic intuitiveness, narrowing the guiding methods into two: "only 
duration" and "rhythm \& duration". These two methods were chosen to be tested with a series of tests, in order to evaluate their learnability, accuracy and their intuitiveness. To both methods an additional vibration ("contra vibration") was added. Both versions, with and without "contra vibration" were tested as well.

\section{PREVIOUS RESEARCH ON VIBROTACTILE GUIDANCE}

The use of vibrotactile feedback as guidance is wide and broadly applied. With the aim of reaching different tasks, numerous applications were developed: as vibrating belts, shoulder pads, dorsal and ventral torso vests, arm and wrists bands. When referring to the tasks of guidance, it is possible to divide them into three categories. Attentional guidance, where vibrotactile feedbacks are used to direct the attention to the location of critical events; movement guidance, where the use of vibrotactile feedbacks is to guide movements and to enhance motor learning and training; and spatial guidance, where vibrotactile stimulation is used directly to guide humans toward a specific target [1].

In the field of aviation various uses of haptic feedback, with the aim of warning the pilot about the onset of a stall and reducing disorientation, were already developed and applied. Devices such as the stick shaker: a stall warning mechanical system, connected to lift detectors and angle of attack sensors, which shakes the control yoke when a stall is imminent [2]. Or such as TSAS - the Tactile Situation Awareness System: the use of a torso harness, fitted with multiple actuators, which can continuously update the pilot's awareness of position [3].

The aim of this project it does not only linger over attentional guidance (i.e. to warn) but it also dwells on spatial and movement guidance (i.e. to guide into a precise way of flying and into solving dangerous flight modes). That is why this work is dedicated to find, to design, to test and to evaluate different guiding methods.

In consequence, a research on the vibrotactile parameters, which can be modified to achieve different guiding methods, follows.

\subsection{Affectible parameters}

From the parameters, which can affect the vibrotactile feedbacks, different motifs can represent simple actions and, when combined, they can represent even more complex concepts. These kinds of connections between the motifs and their meaning require being learned [4]. To interfere more slightly
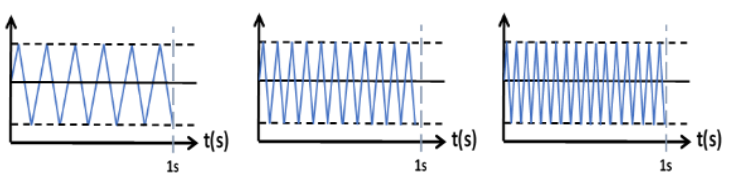

Figure 1: Example of three different frequence's graphs
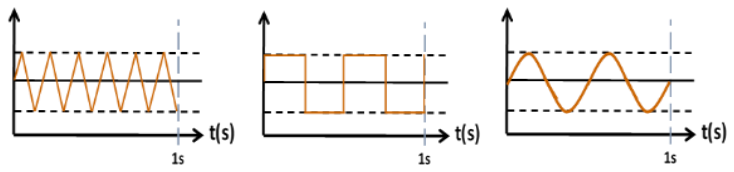

Figure 3: Example of different signal's waveforms
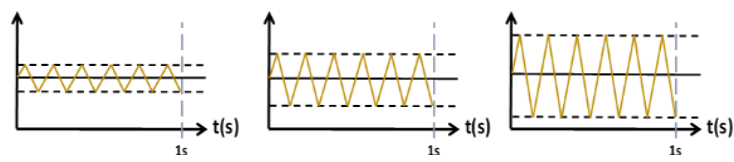

Figure 2: Example of three different amplitude's graphs
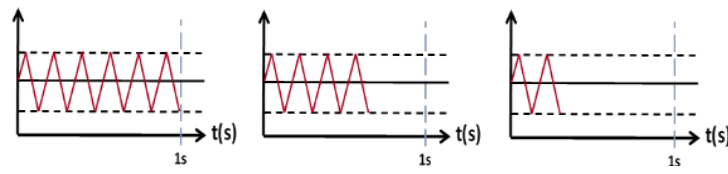

Figure 4: Example of signal's changing duration
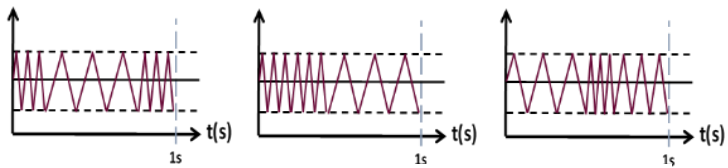

Figure 5: Example of different rhythm paterns 
with the pilot concentration, the reaction of the pilot should be almost automatic. Therefore, one of the aims to achieve is to design guiding methods as simple and as intuitive as possible.

The parameters, which can be manipulated, often vary depending on the type of the actuator. Not all of them allow influencing all the parameters. In general, the tactile affectable parameters are:

- The frequency, which represents the number of cycles per second [5] as shown in Figure 1.

- The amplitude, which defines the strength of the vibration stimuli, which are detected just when the amplitude exceeds a specific threshold [5] - shown in Figure 2.

- The waveform (Figure 3) is a much less important variable because its perception is much more limited. The sine and square waves are possible to differentiate, but slighter differences are almost imperceptible [4].

- When varying the length of a single pulse, we are affecting the duration (Figure 4) of the vibrotactile signal [5].

- The rhythm (Figure 5) represents groups of pulses of different durations which are put in a temporal pattern [5].

- As different locations across the body have different levels of sensitivity and spatial acuity it is possible to use spatially distributed actuators as the variable of body location, which notifies the position of the stimuli [4].

According to [5], the frequency and the amplitude can be treated as one parameter, called intensity, considering that they both change according to the change of voltages, and the change is almost linear.

\section{DEVELOPMENT OF THE METHODS}

Before starting with the designing of different guiding methods, another experiment was done. During this first experiment, with the aim of examining the human reaction on directional vibration impulses, the hardware was made. A joystick representing the control stick, with four vibration engines representing four main directions (forward, backward, left and right), and one vibration engine simulating interferences caused by aircraft vibrations. As results of this experiment the hypothesis that human can distinguish directional vibrations of a control stick was confirmed. A preferred reaction direction (toward the vibration) was evaluated more intuitive and automatic. The interference caused by the fifth vibration engine did not influence results by either objective or subjective evaluation [6].

When the conclusions mentioned above were made, the developing of different guiding methods started. The first step was to define which variables can be affected. Previous research on vibrotactile guidance showed that five parameters can be modified to encode information in general: intensity, waveform, duration, rhythm, and body location. According to [7], it is not possible to modify the waveform of the signal because its manipulation would require specific hardware. Furthermore, in our case, the body location parameter was already used as the perception of the directions.

In summary, guiding can be conducted by altering just three parameters: intensity, rhythm, and duration. Considering that the intensity is a parameter that can be affected in combination with both, rhythm and duration, and particularly considering that its value should be decided in spite of the interferences caused by the aircraft's vibrations, we decided to set this parameter aside and analyze it in the future. Therefore, the focus lingered just on rhythm and duration.

Consequently, the following options are available: "only rhythm", "rhythm \& duration", and "only duration". In order to validate whether all of these options are easy to perceive and to interpret, we investigated them in a first pilot test. To simplify the coding the decision to dwell just with two directions - forward and backward - was taken. 


\subsection{Pilot test}

The pilot test aimed to find out the intuitiveness of the primarily designed guiding methods. The results were supposed to help for a further comparative evaluation. The primary task of each method consisted of following the vibrations to a specific randomly chosen position and trying to hold it. The subject had to perform one trial for each method. Each trial consisted of reaching and holding 21 desired positions (DP). The subject was asked to talk about his perceptions and reactions associated with the vibrotactile feedback.

The movement of the joystick required to achieve the position was toward the direction of the vibrations.

- The "only rhythm" option had five different rhythms varying according to the distance from the DP.

- The "only duration" option consisted of getting shorter vibration pulses the closer the joystick was getting to the DP.

- The "rhythm and duration" option was a combination of both: on further distances, the rhythm patterns were used, but as soon as the joystick reached striking distances to the DP, the "only duration" option took up.

The test aimed to stress out only the intuitiveness of the three different guiding methods mentioned above. Therefore, the average deviation (AE) from the DP, the maximum deviation (ME) from the DP, and the subject's perception were observed.

The subject tested all three options. At the end of the pilot test, the "only rhythm" method was removed. According to the subject's comments, it was too confusing and not accurate enough considering the relatively high speeds the hand achieved on those really small distances.

\subsection{Results and conclusions}

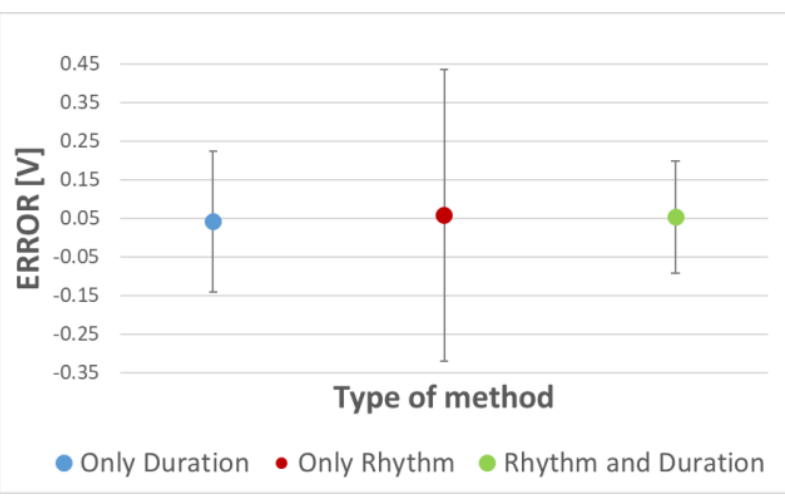

Figure 6: Pilot test - Comparison of the maximum average deviations during each method

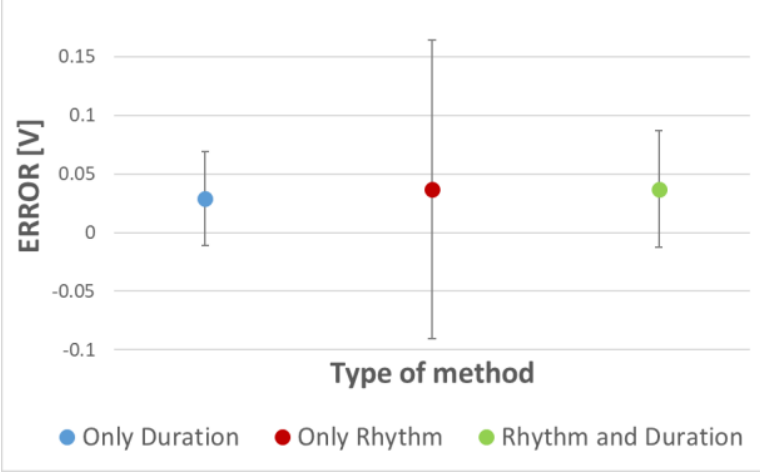

Figure 7: Pilot test - Comparison of the achieved average deviations during each method

Based on the subject assertion, the methods, which were tested, were narrowed into two main guiding methods: the "only duration" and the "rhythm \& duration". This was confirmed by his perception and by a higher ME from the DP and a higher AE measured in the "only rhythm" method as shown in Figure 6 and Figure 7.

With the aim to lower the MEs and the maximum overdrafts of the DP (MODPs), an additional vibration called the "contra vibration" was added to both methods. This vibration occurs only the first time the subject reaches a DP, warning him about it. Both versions, with and without "contra vibration" were tested as well. 


\section{MAIN TESTING}

\subsection{Subjects}

The methods were tested on 22 subjects, 16 males and 6 females in an age range between 21 and 27 . They were randomly divided into two groups - 11 subjects per each method - in order to avoid any alternation during the learning process because of the overlapping of the methods.

\subsection{Hardware}

The hardware used for the experiment included:

- A joystick, representing the control stick of the aircraft. Genius MaxFighter F-16U was the type of the used joystick, and its potentiometers were connected to the microcontroller for position reading,

- Two mobile phone vibration engines (LG Optimus Black P970) representing direction signaling.

- $\quad$ An Arduino MEGA microcontroller.

The vibration engines were placed on the joystick in two directions - forward and backwards - and fastened by four layers of double-sided tape to insulate joystick from spreading vibrations in all directions as shown in Figure 8. The code was run on Arduino MEGA from MatLab environment.

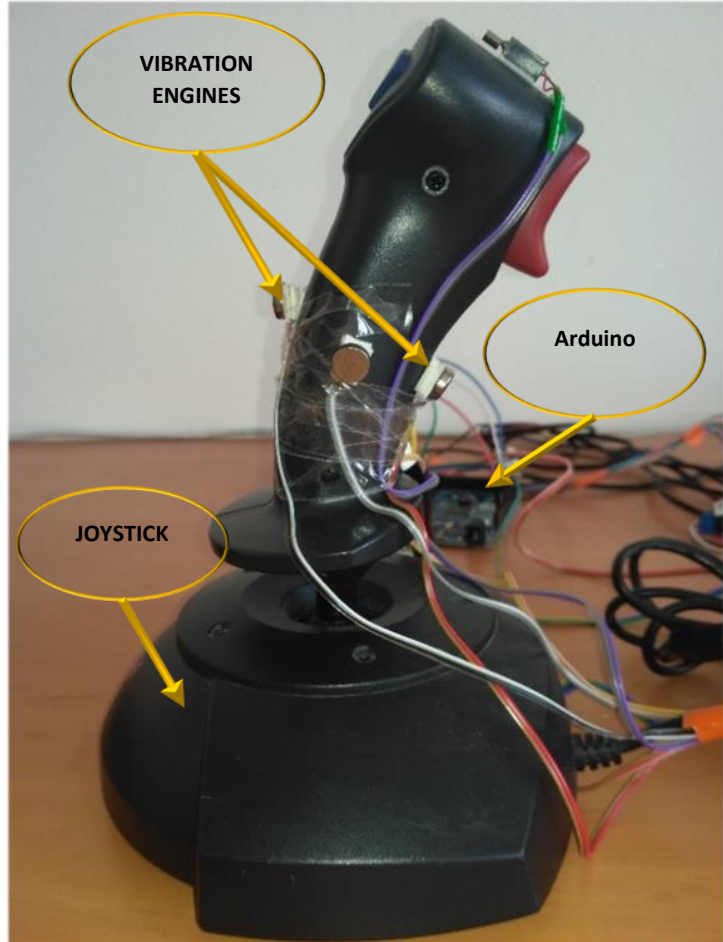

Figure 8: Experimental hardware

\begin{tabular}{|c|c|}
\hline & Definition \\
\hline$H T$ & $\begin{array}{l}\text { The Holding Time represents the total } \\
\text { time in which the subject is able to hold } \\
\text { the DP successfully. }\end{array}$ \\
\hline$L T$ & $\begin{array}{l}\text { The Learning Time represents the time } \\
\text { that the subject needs to hold the } \\
\text { position at least for the first entire } \\
\text { second before he makes any deviations } \\
\text { from the DP. }\end{array}$ \\
\hline$D P T$ & $\begin{array}{c}\text { The Desired Position Time represents all } \\
\text { duration of the DP. Each DP has a } \\
\text { different randomly chosen DPT. }\end{array}$ \\
\hline$A E$ & $\begin{array}{l}\text { The Average Error represents the } \\
\text { average value of the deviations made } \\
\text { when trying to hold the DP. }\end{array}$ \\
\hline$M E$ & $\begin{array}{l}\text { The Maximum Error represents the } \\
\text { maximum value of all the deviations } \\
\text { made when trying to hold each DP. }\end{array}$ \\
\hline MODP & $\begin{array}{l}\text { It represents the maximum value of all } \\
\text { the overdrafts made during the holding } \\
\text { of each DP. The overdraft is the } \\
\text { overcrossing of the DP based on the } \\
\text { initial AP. }\end{array}$ \\
\hline
\end{tabular}

Table 1: Main measured parameter and their definitions

\subsection{Experiment design and procedure}

The testing of each subject consisted of three parts.

1. The LEARNING PART in which the subjects were asked to complete the primary task, which consisted of following the vibrations to a specific randomly chosen position and trying to hold it. They had to reach and hold 30 positions to get accustomed to the method. The measurements of this part, compared with the AFTER-LEARNING PART, were used to evaluate the learnability of the method. 
2. In the AFTER-LEARNING PART, the subjects were asked to complete the same primary task and to reach and hold 30 positions as well. The measurements of this part were compared with the measurements of the same part of the other method. This comparison was used to evaluate the accuracy of the two methods.

3. The measurements made during the CONTRA-VIBRATION PART while reaching and holding $30 \mathrm{DP}$ with the addition of the "contra-vibration" were compared to the measurements made during the AFTER-LEARNING PART. In this way, they were used to evaluate the usefulness of the "contra vibration".

All the subjects were asked to tell their perceptions.

Because of the variance of the response, due to the changing of the participants' attention, the haptic sense was isolated by putting headphones with calm instrumental music on each subject, preventing them from relying on combining the haptic sense with the audible sensing of the vibrations.

The main parameters on which the evaluation was done are explained in Table 1.

\subsection{Guiding methods}

The explanation of the design of the two guiding methods follows.

The "only duration" method - when a position is chosen, a periodic vibration points the direction in which to move the joystick - i.e. toward the vibrations. The closer the joystick's actual position (AP) gets to the DP, the shorter the pulse's duration gets.
The "rhythm and duration" method - when a position is chosen, a periodic vibration points the direction in which to move the joystick -i.e. toward the vibrations. The rhythm of the vibrations changes in accordance with the distance between the AP and the DP. There are 3 different rhythms connected to this distance (»Far distance», "Closer distance», " Striking distance" - shown in Figure 9). When the joystick is in the "Striking distance", the vibration gets in the same modality as in the "only duration « method: the closer the joystick's AP gets to the DP, the shorter the pulse's duration gets.

For both methods - when the DP is reached, there are no more vibrations. The same is applied when the position is held successfully. If the joystick is moved from the DP, a periodic vibration points the direction in which to make the correction. The pulse's length depends on the distance between the DP and the AP. The subject's main task is to reach and hold the position as precisely as possible until the next one is not chosen and pointed by the vibrations.

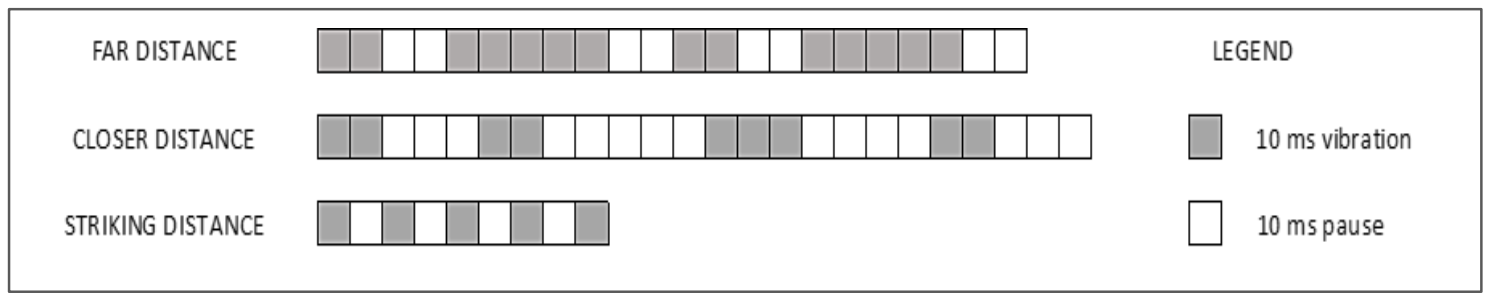

Figure 9: Rhythm patterns of the »rhythm\&duration« method. 


\section{EVALUATION AND RESULTS}

Based on the measured parameters a series of hypothesis tests were conducted to evaluate the accuracy of each method, the usefulness of the "contra vibration" and the learnability of each method.

\subsection{The accuracy of the methods}

\begin{tabular}{|l|c|c|}
\hline \multicolumn{3}{|c|}{ Ho: $\mu_{\text {AEs_OnlyDuration }-} \mu_{\text {AEs_Rhythm\&Duration }=0}$} \\
\hline \multicolumn{3}{|c|}{ Ha: $\mu_{\text {AEs_OnlyDuration }-} \mu_{\text {AEs_Rhythm\&Duration > } 0}$} \\
\hline & AEs_OD & AEs_R\&D \\
\hline Mean [V] & 0.065 & 0.079 \\
\hline Variance [V] & 0.0022 & 0.0034 \\
\hline Observations [/] & 330 & 330 \\
\hline Hypothesized Mean Difference & \multicolumn{2}{|c|}{0} \\
\hline df & \multicolumn{2}{|c|}{3.497} \\
\hline t Stat & \multicolumn{2}{|c|}{0.00025} \\
\hline P(T<=t) one-tail & \multicolumn{2}{|c|}{1.647} \\
\hline t Critical one-tail & \multicolumn{2}{|c|}{} \\
\hline
\end{tabular}

Table 2: Results of the t-test made with the comparison of the AEs achieved by the subjects during the AFTERLEARNING PART

\begin{tabular}{|c|c|c|}
\hline \multicolumn{3}{|c|}{ 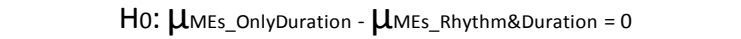 } \\
\hline \multicolumn{3}{|c|}{ 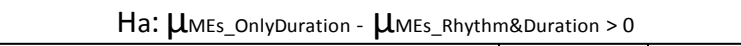 } \\
\hline & MEs_R\&D & MEs_OD \\
\hline Mean [V] & 0.228 & 0.181 \\
\hline Variance [V] & 0.0679 & 0.0449 \\
\hline Observations [/] & 330 & 330 \\
\hline Hypothesized Mean Difference & \multicolumn{2}{|c|}{0} \\
\hline df & \multicolumn{2}{|c|}{632} \\
\hline t Stat & \multicolumn{2}{|c|}{2.578} \\
\hline $\mathrm{P}(\mathrm{T}<=\mathrm{t})$ one-tail & \multicolumn{2}{|c|}{0.0051} \\
\hline t Critical one-tail & \multicolumn{2}{|c|}{1.647} \\
\hline
\end{tabular}

Table 3: Results of the t-test made with the comparison of the MEs achieved by the subjects during the AFTERLEARNING PART

\begin{tabular}{|c|c|c|}
\hline \multicolumn{3}{|c|}{ 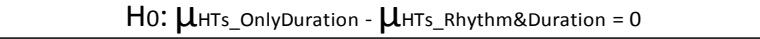 } \\
\hline \multicolumn{3}{|c|}{ 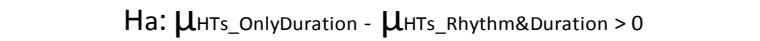 } \\
\hline & HTs_OD & HTs_R\&D \\
\hline Mean [s] & 10.49 & 8.53 \\
\hline Variance [s] & 32.85 & 28.63 \\
\hline Observations [/] & 330 & 330 \\
\hline Hypothesized Mean Difference & \multicolumn{2}{|c|}{0} \\
\hline df & \multicolumn{2}{|c|}{655} \\
\hline t Stat & \multicolumn{2}{|c|}{4.55} \\
\hline $\mathrm{P}(\mathrm{T}<=\mathrm{t})$ one-tail & \multicolumn{2}{|c|}{0.000003} \\
\hline t Critical one-tail & \multicolumn{2}{|c|}{1.647} \\
\hline
\end{tabular}

Table 4: Results of the t-test made with the comparison of the HTs achieved by the subjects during the AFTERLEARNING PART

In order to evaluate the accuracy of the methods, three two-sample one-tailed t-tests with a level of confidence of $95 \%$ were performed. Each of them is comparing the two methods through one of the parameters (AEs, MEs and HTs) achieved by the subjects during the AFTER-LEARNING PART.

For each test the three conditions for valid t-intervals were reached:

- The data was a random sample from the population of interest,

- The sampling distribution of the sample was approximately normal ( $n^{\circ}$ of measurements $\geq 30$ ),

- The individual observations can be considered independent.

1. The AEs achieved by the "only duration" method subjects were significantly lower $(P=0.00025)$ than those achieved by the "rhythm \& duration" method subjects. The results of the test are shown in Table 2. Figure 10 is an example of the frequency of the measured AEs as an approximately normal distribution.

2. The MEs achieved by the "only duration" method subjects were significantly lower $(P=0.0051)$ than those achieved by the "rhythm \& duration" method subjects. The results of the test are shown in Table 3.

3. The HTs achieved by the "only duration" method subjects were significantly higher $(P=0.000003)$ than those achieved by the "rhythm \& duration" method subjects. The results of the test are shown in Table 4. 
In conclusion, all three tests asserted that the "only duration" method is significantly more accurate than the "rhythm \& duration" method, with lower achieved AEs and MEs, and with higher achieved HTs.

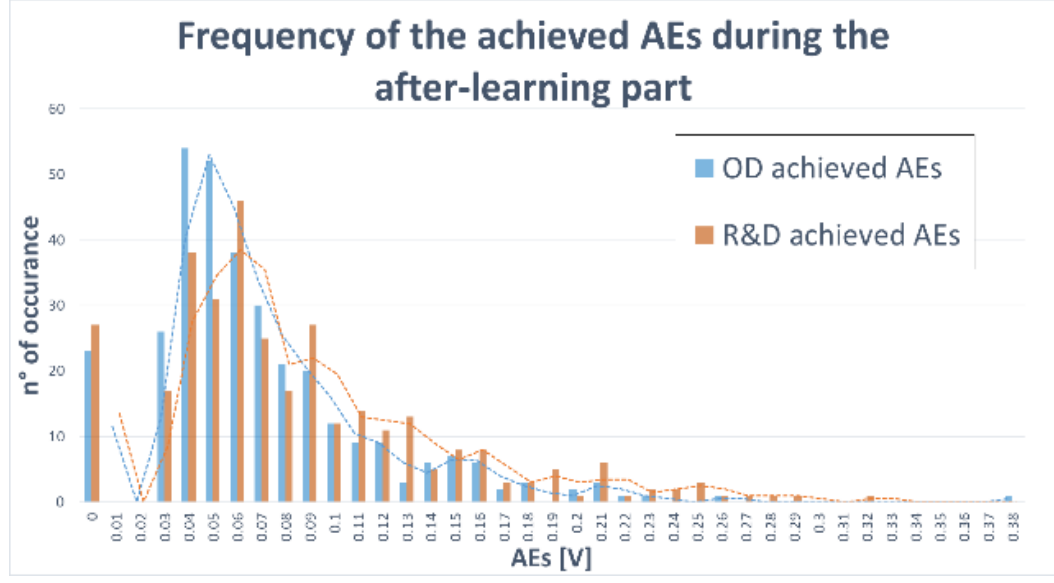

Figure 10: The Frequency of the measured AEs during the AFTER-LEARNING PART

\subsection{The usefulness of the "contra vibration"}

To evaluate the effects of "the contra vibration" three one-tailed paired t-test conducted with a confidence level of $95 \%$ were performed for each method. Each of them compared parameters (AEs, MEs and MODPs) achieved by the subjects during the AFTER-LEARNING PART and the CONTRAVIBRATION PART. For each test, the three conditions for valid t-intervals were reached.

\section{Assertions for the "only duration" method}

1. The difference between the achieved AEs during the CONTRA-VIBRATION PART and the AFTERLEARNING PART of the testing was not significant enough $(P=0,264)$ to assert that there is an improvement thanks to the addition of the "contra vibration«. The results of the test are shown in Table 5.

2. The difference between the achieved MEs during the CONTRA-VIBRATION PART and the AFTERLEARNING PART of the testing was not significant enough $(P=0,315)$ to assert that there is an improvement thanks to the addition of the contra vibration. The results of the test are shown in Table 6.

3. The difference between the achieved MODPs during the CONTRA-VIBRATION PART and AFTERLEARNING PART of the testing was not significant enough $(P=0,283)$ to assert that there is an improvement thanks to the addition of the contra vibration. The results of the test are shown in Table 7.

Assertions for the "rhythm \& duration" method

1. The AEs achieved during the CONTRA-VIBRATION PART are significantly higher $(P=0,0000012)$ than those achieved during the AFTER-LEARNING PART. The results of the test are shown in Table 8.

2. The MEs achieved during the CONTRA-VIBRATION PART are significantly higher $(P=0,000013)$ than those achieved during the AFTER-LEARNING PART. The results of the test are shown in Table 9.

3. The MODPs achieved during the CONTRA-VIBRATION PART are significantly higher $(P=0,0097)$ than those achieved during the AFTER-LEARNING PART. The results of the test are shown in Table 10. 
In conclusion, the tests performed on the "only duration" method asserted that the addition of the "contra vibration" had not led to any significant improvement to any of the measured parameters.

Those performed on the "rhythm \& duration" method asserted that the addition of the "contra vibration" led to a significant decrease of the accuracy affecting all three parameters negatively.

Beside it, 18 subjects out of 22 affirmed that the "contra vibration" strongly confused them because of a sudden additional vibration that made them react with additional movements and less accuracy. Based on their assertions the "contra vibration" is confusing and not that much intuitive.

\begin{tabular}{|l|c|c|}
\hline \multicolumn{3}{|c|}{ Ho: $\mu_{\text {AEs_NONCONTRA - }} \mu_{\text {AEs_CONTRA }=0}$} \\
\hline \multicolumn{3}{|c|}{ Ha: $\mu_{\text {AEs_NONCONTRA - }} \mu_{\text {AEs_CONTRA > } 0}$} \\
\hline Mean [V] & AEs_NONC & AEs_C \\
\hline Variance [V] & 0.065 & 0.067 \\
\hline Observations [/] & 0.0022 & 0.0033 \\
\hline Hypothesized Mean Difference & 330 & 330 \\
\hline df & \multicolumn{2}{|c|}{0} \\
\hline t Stat & \multicolumn{2}{|c|}{329} \\
\hline P(T<=t) one-tail & \multicolumn{2}{|c|}{0.6302} \\
\hline $\mathbf{t}$ Critical one-tail & \multicolumn{2}{|c|}{1.967} \\
\hline
\end{tabular}

Table 5: "Only duration« method - Results of the t-test made with the comparison of the AEs achieved by the subjects during the AFTER-LEARNING PART and the CONTRA-VIBRATION PART

\begin{tabular}{|c|c|c|}
\hline \multicolumn{3}{|c|}{ 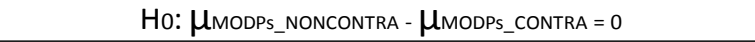 } \\
\hline \multicolumn{3}{|c|}{ 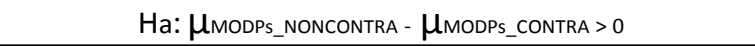 } \\
\hline & MODPs_NONC & MODPs_C \\
\hline Mean [V] & 0.156 & 0.147 \\
\hline Variance [V] & 0.04 & 0.042 \\
\hline Observations [/] & 330 & 330 \\
\hline Hypothesized Mean Difference & \multicolumn{2}{|l|}{0} \\
\hline df & \multicolumn{2}{|c|}{329} \\
\hline t Stat & \multicolumn{2}{|c|}{0.574} \\
\hline$P(T<=t)$ one-tail & \multicolumn{2}{|c|}{0.283} \\
\hline t Critical one-tail & \multicolumn{2}{|c|}{1.649} \\
\hline
\end{tabular}

Table 7: "Only duration« method - Results of the t-test made with the comparison of the MODPs achieved by the subjects during the AFTER-LEARNING PART and the CONTRA-VIBRATION PART

\begin{tabular}{|c|c|c|}
\hline \multicolumn{3}{|c|}{ 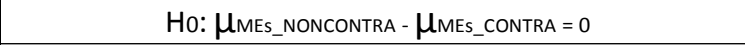 } \\
\hline \multicolumn{3}{|c|}{ Ha: $\mu_{\text {MEs_NONCONTRA - }} \mu_{\text {MEs_CONTRA }}>0$} \\
\hline & MEs_NONC & MEs_c \\
\hline Mean [V] & 0.228425749 & 0.336433 \\
\hline Variance $[\mathrm{V}]$ & 0.067927032 & 0.166388 \\
\hline Observations [/] & 330 & 330 \\
\hline Hypothesized Mean Difference & 0 & \\
\hline df & 329 & \\
\hline$t$ Stat & -4.26 & \\
\hline $\mathrm{P}(\mathrm{T}<=\mathrm{t})$ one-tail & 0.0000 & \\
\hline t Critical one-tail & $1.64 \mathrm{~S}$ & \\
\hline
\end{tabular}

Table 9: "Rhythm\&duration « method - Results of the t-test made with the comparison of the MEs achieved by the subjects during the AFTER-LEARNING PART and the CONTRA-VIBRATION PART

\begin{tabular}{|c|c|c|}
\hline \multicolumn{3}{|c|}{ 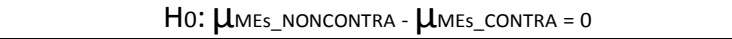 } \\
\hline \multicolumn{3}{|c|}{ 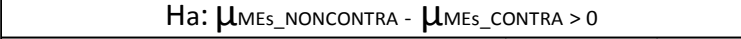 } \\
\hline & MEs_NONC & MEs_C \\
\hline Mean [V] & 0.177 & 0.185 \\
\hline Variance [V] & 0.041 & 0.062 \\
\hline Observations [/] & 330 & 330 \\
\hline Hypothesized Mean Difference & \multicolumn{2}{|l|}{0} \\
\hline df & \multicolumn{2}{|c|}{329} \\
\hline t Stat & \multicolumn{2}{|c|}{-0.482} \\
\hline$P(T<=t)$ one-tail & \multicolumn{2}{|c|}{0.315} \\
\hline t Critical one-tail & \multicolumn{2}{|c|}{1.649} \\
\hline
\end{tabular}

Table 6: "Only duration« method - Results of the t-test made with the comparison of the MEs achieved by the subjects during the AFTER-LEARNING PART and the CONTRA-VIBRATION PART

\begin{tabular}{|c|c|c|}
\hline \multicolumn{3}{|c|}{ Ho: $\mu_{\text {AEs_NONCONTRA }-} \mu_{\text {AEs_CONTRA }}=0$} \\
\hline \multicolumn{3}{|c|}{ Ha: $\mu_{\text {AEs_NONCONTRA }-} \mu_{\text {AEs_CONTRA }>0}$} \\
\hline & AEs_NONC & AEs_C \\
\hline Mean [V] & 0.079243711 & 0.106181 \\
\hline Variance [V] & 0.003396168 & 0.007194 \\
\hline Observations [/] & 330 & 330 \\
\hline Hypothesized Mean Difference & 0 & \\
\hline df & 329 & \\
\hline t Stat & -4.801 & \\
\hline$P(T<=t)$ one-tail & 0.00000 & \\
\hline t Critical one-tail & 1.649 & \\
\hline
\end{tabular}

Table 8: "Rhythm\&duration « method - Results of the ttest made with the comparison of the AEs achieved by the subjects during the AFTER-LEARNING PART and the CONTRA-VIBRATION PART

\begin{tabular}{|c|c|c|}
\hline \multicolumn{3}{|c|}{ 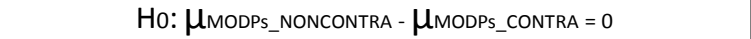 } \\
\hline \multicolumn{3}{|c|}{ 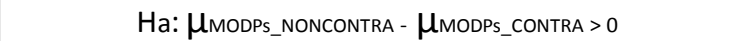 } \\
\hline & MODPs_NONC & MODPs_C \\
\hline Mean [V] & 0.199561585 & 0.255134 \\
\hline Variance [V] & 0.067717423 & 0.126208 \\
\hline Observations [/] & 330 & 330 \\
\hline Hypothesized Mean Difference & \multicolumn{2}{|l|}{0} \\
\hline df & \multicolumn{2}{|l|}{329} \\
\hline t Stat & \multicolumn{2}{|c|}{-2.345} \\
\hline $\mathrm{P}(\mathrm{T}<=\mathrm{t})$ one-tail & \multicolumn{2}{|c|}{0.0097} \\
\hline t Critical one-tail & \multicolumn{2}{|c|}{1.649} \\
\hline
\end{tabular}

Table 10: "Rhythm\&duration « method - Results of the ttest made with the comparison of the MODPs achieved by the subjects during the AFTER-LEARNING PART and the CONTRA-VIBRATION PART 


\subsection{The learnability of the methods}

To evaluate the learnability of each method the ratios between the HTs and the DPTs achieved during the LEARNING PART were compared with those achieved during the AFTER-LEARNING PART. This was done separately for each subject with a one-tailed paired t-test with a level of confidence of $95 \%$.

The same comparison was performed with the LTs parameter.

For each test, the three conditions for valid t intervals were reached.

Only four subjects out of eleven tested on the "only duration" method reached a significant improvement in the HT ratios ( $P$-values $-0.00019,0.015,0.0034,0.042$ ) and a significant improvement in the LTs (P-values $-0.013,0.031,0.031,0.0004)$.

Whereas nine subjects out of eleven tested on the "rhythm \& duration" method reached a significant improvement in the HT ratios (P-values - 0.0083, 0.046, 0.0073, 0.0018, 0.0019, 0.048, 0.000004, $0.00028,0.0159)$, and seven out of eleven reached a significant improvement in the LTs (P-values $0.003,0.016,0.029,0.007,0.0001,0.0003,0.026)$.

In conclusion, it is possible to assert that the "only duration « method achieved fewer improvements during the learning process than the "rhythm \& duration « method. This can be explaned taking in consideration the previously done conclusions: the "only duration « method achieved a greater accuracy from the subjects, that's why the improvements done from the LEARNING PART to the AFTERLEARNING PART are significantly smaller, unperceivable or inexistent.

\section{CONCLUSIONS}

After defining the parameters which can be manipulated, three different affectable combinations where designed: "duration only", "rhythm only" and "rhythm \& duration". The pilot test narrowed them into two - "duration only" and "rhythm \& duration". To both methods the "contra vibration" was added, with the aim of increasing the accuracy. The two methods and the "contra vibration" were tested with a series of hypothesis tests. Based on the results:

- The "only duration" method was defined significantly more accurate than the "rhythm \& duration" method;

- The "contra vibration" led to no significant improvements for the "only duration" method and a significant decrease in the accuracy of the "rhythm \& duration" method;

- The "rhythm \& duration" method achieved greater improvement during the learning process than the "only duration" method.

\section{ACKNOWLEDGEMENTS}

This research was partially supported by TACR, project no. TJ01000122 "Haptic feedback of assistant systems for flight safety improvement" and by the Erasmus+ programme of the European Union. 


\section{REFERENCES}

[1] WEBER, Bernhard, et al. Evaluation of a vibrotactile feedback device for spatial guidance. In: World Haptics Conference (WHC), 2011 IEEE. IEEE, 2011. p. 349-354.

[2] GAST, Mark E. Autopilot/flight director stall protection system. U.S. Patent No 5,803,408, 1998.

[3] RUPERT, Angus H. An instrumentation solution for reducing spatial disorientation mishaps. IEEE Engineering in Medicine and Biology Magazine, 2000, 19.2: 71-80.

[4] BREWSTER, Stephen; BROWN, Lorna M. Tactons: structured tactile messages for non-visual information display. In: Proceedings of the fifth conference on Australasian user interface-Volume 28. Australian Computer Society, Inc., 2004. p. 15-23.

[5] ASIF, Amna; HEUTEN, Wilko; BOLL, Susanne. Exploring distance encodings with a tactile display to convey turn by turn information in automobiles. In: Proceedings of the 6th Nordic conference on human-computer interaction: Extending Boundaries. ACM, 2010. p. 32-41.

[6] ZIKMUND, Pavel; MACIK, Miroslav; MIKOVEC, Zdenek; Reaction on directional vibrations applied on joystick, New Trends in Civil Aviation 2017, will be published in 2018.

[7] VAN ERP, Jan BF. Guidelines for the use of vibro-tactile displays in human-computer interaction. In: Proceedings of eurohaptics. 2002. p. 18-22.

\section{COPYRIGHT STATEMENT}

The authors confirm that they, and/or their company or organization, hold copyright on all of the original material included in this paper. The authors also confirm that they have obtained permission, from the copyright holder of any third party material included in this paper, to publish it as part of their paper. The authors confirm that they give permission, or have obtained permission from the copyright holder of this paper, for the publication and distribution of this paper as part of the READ 2018 proceedings.

This is an open access article distributed under the Creative Commons Attribution License which permits unrestricted use, distribution, and reproduction in any medium, provided the original work is properly cited. (CC BY 4.0). 